\title{
Schwannoma of the median nerve mimicking carpal tunnel syndrome in a pregnant patient. Case report.
}

\author{
Giokits-Kakavouli Giasna ${ }^{1,2}$, Micu C. Mihaela ${ }^{3}$, Micu Romeo ${ }^{4}$
}

${ }^{1}$ Department of Sports Medicine and physiology, University of Medicine, 69 Svetozara Markovica Street, 34000 Kragujevac, Serbia, ${ }^{2}$ Private Office, 10 Solomou Street, 60132 Katerini, Greece, ${ }^{3}$ Rheumatology Division, $2^{\text {nd }}$ Rehabilitation Department, Clinical Rehabilitation Hospital Cluj- Napoca, Romania, ${ }^{4}$ Assisted Reproduction Department, Gynecology I Clinic Cluj- Napoca, "Iuliu Hatieganu” University of Medicine and Pharmacy Cluj- Napoca, Romania

\begin{abstract}
In patients with symptoms of a peripheral neuropathy especially during pregnancy, use of imaging techniques such as Ultrasound (US) and Magnetic Resonance Imaging (MRI) may be essential for the diagnostic accomplishment. A 30-weekspregnant diabetic female attending US evaluation due to intermittent hand pain, numbness, and weakness bilaterally. Although, the US evaluation revealed the median nerve (MN) normal size, echogenicity and echo-texture within the right carpal tunnel, the US assessment applied proximally to the carpal tunnel, revealed a hypoechoic tumor-like mass and increased MN cross section area. In transverse view, the MN was detected as an eccentric, hypoechoic structure compressed by the aforementioned mass. A presence of MN schwannoma or neurofibroma was suspected. US has been proved to be extremely useful to determine location, extent as well as the type of nerve lesion.
\end{abstract}

Keywords: median nerve, schwannoma, ultrasound, carpal tunnel syndrome, pregnancy.

\section{Introduction}

Traumatic, inflammatory, autoimmune, and idiopathic conditions may cause derangement of peripheral nerves. Carpal tunnel syndrome (CTS) is the most common entrapment syndrome and may be caused by any kind of median nerve $(\mathrm{MN})$ compression at the wrist level. Some anatomical variants such as a presence of accessory muscles or bifid MN, as well as the presence of space-occupying lesions within the carpal tunnel (ganglion cysts and tumors), may be predisposing factors related to CTS. Some endocrine disorders (diabetes, hypothyroidism, and acromegalia) or systemic storage diseases (amyloidosis and mucopolysacharidosis) may produce a $\mathrm{MN}$ compression due to pathologic deposition

Received 05.06.2016 Accepted 14.07.2016

Med Ultrason

2016, Vol. 18, No 4, 521-523

Corresponding author: Micu Romeo

Assisted Reproduction Department, Gynecology I Clinic

"Iuliu Hatieganu" University of Medicine and

Pharmacy, Cluj- Napoca, Romania

E-mail: romeomicu@hotmail.com of various substances within the tunnel. However, some normal conditions such as pregnancy, especially in the $3^{\text {rd }}$ trimester, can cause similar symptoms.

MRI is currently the most preferable method in the visualization of peripheral nerves but due to the continuous improvement of US method, it has been established as a valuable tool in the management of the peripheral nerves' pathology $[1,2]$. Using the high-end machines it is possible to demonstrate even the smallest distal nerve branches [3-5]. In compare with advanced imaging techniques such as CT and MRI it is a very easy, quick, painless and efficient method for diagnostic as well as for the treatment purposes (aspirations, injections).

\section{Case report}

A 39-year-old, 30-weeks-pregnant female visited the rheumatology department, complaining of intermittent aching sensation over the ventral wrist aspect as well as with the first three digits bilaterally. The same pattern numbness was aggravated during the night, as well as the grip weakness was mostly expressed in the non-dominant hand (left). During the last 3 weeks, symptoms had be- 
come intense and continuous, affecting daily activities as well as the night sleep. The patient was diagnosed with diabetes mellitus (DM) 10 years ago and 4 years ago, during the first pregnancy, she experienced the same symptoms in the left hand. She had been clinically diagnosed with left CTS and the symptoms remitted after the local corticosteroid (CS) injection.

Currently, after the clinical examination, the same interventional procedure was repeated; this time, in both hands. Insulin therapy was adjusted for the next days in accordance with glycemic blood levels. However, no remarkable results were reported in the right hand. The patient was relegated for US evaluation of the carpal tunnel, with a possibility of a second US-guided CT injection, if considered necessary.

The right hand examination revealed a small lump located in the proximity of the flexor digitorum tendons, $2 \mathrm{~cm}$ proximal to the wrist. Hoffmann-Tinel's and Phallen's tests were both negative. The 20-second-transducer compression to the carpal tunnel entrance level, triggered numbness at the area of the left median nerve (MN) supply. The test was negative in the right hand.

US evaluation revealed the MN normal size, echo-texture, and echogenicity in the right hand, obtained in two orthogonal planes. At $2 \mathrm{~cm}$ proximally to the carpal tunnel entrance, an abrupt increase of the MN cross section area was identified. The characteristic fascicular shape was replaced by a hypo-echoic tumor like mass of $18.3 \times 6.2$ $\mathrm{mm}$, displaying a Doppler signal. In transverse view, the $\mathrm{MN}$ was detectable as an eccentric, mostly hypoechoic structure compressed by the formerly described mass (fig 1a,b). A suspicion of MN schwannoma was raised.

Left hand US evaluation identified a bifid MN with an increased cross sectional area (sum of the 2 cross sectional areas of $15 \mathrm{~mm}^{2}$ ) and the persistence of the median artery. In longitudinal view, compression of the MN under the flexor retinaculum ("notch sign") and an enlargement of the diameter proximal to the carpal tunnel were identified (fig 1f,g).

After delivery, contrast MRI was performed and a fusiform, well defined, small, inhomogeneous MN tumor $(18 \times 9.7 \mathrm{~mm})$ expressing T2 hyper- signal, T1 iso-signal with the surrounding muscles (flexor carpi radialis, flexor pollicis longus, superficial and deep digitorum flexors) and contrast substance load, suggestive for a schwannoma, was identified (fig 1d,e). The patient postponed surgical treatment.

A year after, during the follow up, US examination revealed the same aspect of the schwannoma. The patient presented no symptoms in the right hand. In the left one, intermittent symptoms after long working days were described.

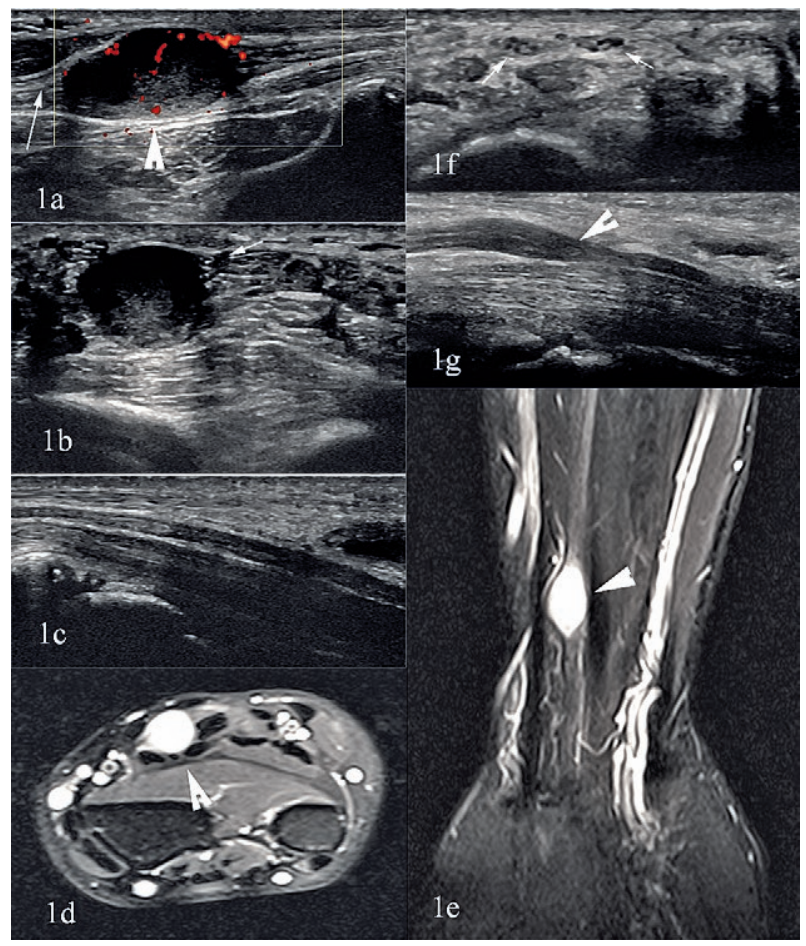

Fig 1. a) Longitudinal grey scale and Doppler mode scanning proximal to the right carpal tunnel. Arrow - normal fascicular $\mathrm{MN}$, arrow head- hypoechoic tumor like mass, positive Doppler signal; b). Transverse grey scale scanning proximal to the right carpal tunnel. Arrow - MN with normal "honeycomb" structure compressed and located eccentric to the hypoechoic mass; c) Longitudinal grey scale scanning of the MN within the right carpal tunnel. Normal aspect of the MN; d) MRI transverse view proximal to the right carpal tunnel. Arrowhead - MN schwannoma; e) MRI- longitudinal view proximal to the right carpal tunnel. Arrowhead - MN schwannoma: f) Transverse grey scale scanning of the MN in the left carpal tunnel showing a bifid nerve (arrows) and the presence of the persistent median artery: g) Longitudinal grey scale scanning of the $\mathrm{MN}$ in the left carpal tunnel showing a swollen $\mathrm{MN}$ proximal to the carpal tunnel, 'notch sign' (arrow head).

\section{Discussions}

The CTS is associated with different demographic and genetic factors as well as with certain medical/ physiological conditions and working habits [7-10]. In this case, the female gender, $3^{\text {rd }}$ trimester pregnancy at the end of the fourth decade, DM, anatomic variant of the $\mathrm{MN}$, and previous CTS history were significant factors contributing to the development of the clinical picture. The bifid MN and median artery presence per se, exposes itself to CTS development. In conjunction with a pregnancy and DM it can trigger chronic MN compression symptoms. The "notch sign" presence supports the hypothesis of a chronic pathology that should be regularly 
checked out. In this case, a local CS injection was shown to be effective.

Schwannoma is the most common benign tumor (arising from the Schwann cells in the nerve sheath) of the peripheral nerves. The MN is one of the most affected peripheral nerves. Usually schwannomas are painless, but in some cases they became symptomatic (pain, paresthesias and motor weakness) [11].

It is well known that there is limitations in performing certain imaging or electrophysiological investigations in the case of non-life threatening conditions during pregnancy. In fact, certain medication protocols (NSAIDs) are restricted mostly after 30-32 weeks of pregnancy due to fetal ( $1^{\text {st }}$ trimester- higher rates of spontaneous abortion, $2^{\text {nd }}$ and $3^{\text {rd }}$ trimester- premature closure of the arterial duct and risk for subsequent pulmonary hypertension development) and maternal (excessive bleeding during delivery) possible side effects $[12,13]$. Moreover, in a pregnancy associated with DM, medication with impact on the kidney function and glycemic levels should be given with caution.

In this particular case, US detected a different NM pathology, outside the right carpal tunnel but, mimicking a true CTS. Unnecessary maternal and fetal exposure to CS was avoided. US, electromyography, and MRI are useful tools complementary to the clinical examination. US detects the tumor but the clear depiction and differentiation may be supported by MRI assessment [10]. Surgical removal is usually curative. However, some authors recommend it only in symptomatic tumors with rapid enlargement or symptoms presentation during the follow-up.

US can be very useful in detecting local causes of MN compression inside the carpal tunnel. This information may change the therapeutic approach. Of note, US has advantages over MRI assessment, being totally safe in pregnancy, showing a higher spatial resolution and allowing the evaluation of long nerve segments in a short time and in a comparative fashion $[14,15]$.

\section{References}

1. Klauser AS, Faschingbauer R, Bauer T, et al. Entrapment neuropathies II: carpal tunnel syndrome. Semin Musculoskelet Radiol 2010;14:487-500.
2. Moller I, Bong D, De Miguel E. Soft Tissue Rheumatism. In: Wakefield R ed. Essential Applications of Musculoskeletal Ultrasound in Rheumatology. Philaelphia, PA: Saunders Elsevier, 2010:219-234.

3. Lucchetta M, Liotta GA, Briani C, et al. Ultrasound diagnosis of peroneal nerve variant in a child with compressive mononeuropathy. J Pediatr Surg 2011;46:405-407.

4. Tagliafico A, Perez MM, Padua L, Klauser A, Zicca A, Martinoli C. Increased reflectivity and loss in bulk of the pronator quadratus muscle does not always indicate anterior interosseous neuropathy on ultrasound. Eur J Radiol 2013;82:526-529.

5. Smith J, Rizzo M, Finnoff JT, Sayeed YA, Michaud J, Martinoli C. Sonographic appearance of the posterior interosseous nerve at the wrist. J Ultrasound Med 2011;30:12331239.

6. Harder A, Wesemann M, Hagel C, et al. Hybrid neurofibroma/schwannoma is overrepresented among schwannomatosis and neurofibromatosis patients. Am J Surg Pathol 2012;36:702-709.

7. Kao SY. Carpal tunnel syndrome as an occupational disease. J Am Board Fam Pract 2003; 16:533-542.

8. Buchberger W, Judmaier W, Birbamer G, Lener M, Schmidauer C. Carpal tunnel syndrome: diagnosis with high-resolution sonography. AJR Am J Roentgenol 1992;159:793-798.

9. El Miedany Y, Ashour S, Youssef S, Mehanna A, Meky FA. Clinical diagnosis of carpal tunnel syndrome: old tests-new concepts. Joint Bone Spine 2008;75:451-457.

10. Klauser AS, Halpern EJ, De Zordo T, et al. Carpal tunnel syndrome assessment with US: value of additional crosssectional area measurements of the median nerve in patients versus healthy volunteers. Radiology 2009;250:171-177.

11. Ozdemir O, Ozsoy MH, Kurt C, Coskunol E, Calli I. Schwannomas of the hand and wrist: long-term results and review of the literature. J Orthop Surg (Hong Kong) 2005; 13:267-272.

12. Koren G, Florescu A, Costei AM, Boskovic R, Moretti ME. Nonsteroidal antiinflammatory drugs during third trimester and the risk of premature closure of the ductus arteriosus: a meta-analysis. Ann Pharmacother 2006;40:824-829.

13. Nakhai-Pour HR, Broy P, Sheehy O, Berard A. Use of nonaspirin nonsteroidal anti-inflammatory drugs during pregnancy and the risk of spontaneous abortion. CMAJ 2011;183:1713-1720.

14. Bruyn GA, Moller I, Klauser A, Martinoli C. Soft tissue pathology: regional pain syndromes, nerves and ligaments. Rheumatology (Oxford) 2012;51 Suppl 7:vii22-vii25.

15. Martinoli C, Bianchi S, Dahmane M, Pugliese F, BianchiZamorani MP, Valle M. Ultrasound of tendons and nerves. Eur Radiol 2002;12:44-55. 